\title{
After Capture: Fundamentals of Images, their Storage, Visualization, Processing and Quantification
}

\author{
Sam A. Johnson ${ }^{1}$ \\ ${ }^{1}$ Light Microscopy Core Facility, Duke University, Durham, NC, USA
}

A diverse array of modern microscopy methods can produce image data in many forms, formats and quantities. Accurate and efficient handling of these raw data and the underlying information contained within is an essential task and one that grows in importance as the volume, complexity and ubiquity of images used in scientific research and development continues to expand.

This presentation will provide an overview of the fundamentals of images and how to efficiently and faithfully deal with them. What can and should be done with these images? What shouldn't be done? How are complex image sets best visualized? How can quantitative values be extracted from images? These topics will be covered with some real-world examples providing background understanding and practical tips for establishing a work-flow to allow you to get the most from your data.

Images produced using scientific instrumentation are often stored in specific formats and have characteristics unlike "ordinary" images. Additional dimensions of data maybe be present, the range of intensity values is often larger in scientific images and the dynamic range may not be completely filled. These need to be understood and acted upon to accurately view and appreciate the data in images and avoid accidental misinterpretation, misrepresentation and quantitative changes.

The size of image data involved in a study varies by many orders of magnitude and large data volumes can present significant problems for file transfer, storage and processing. Differing solutions to the optimal storage and access of data exist for varying scales of data size. Automation of any processing or analysis is essential for operations on significant numbers of images.

The inherent multi-dimensional nature of many image datasets present experimental opportunity and some data challenges. Different methods of assembly and visualization of data exist and the optimal choice differs according to the sample and aim. Projections are among the most commonly used types of $3 \mathrm{D}$ visualization.

Image processing tools offer many possibilities for modifying image data. Can the raw images be enhanced? Should they be visually improved? Many tools are available and an appreciation of their underlying principles and methods of action is vital for ensuring correct and full usage.

Images are a visual display of an array of intensity values. Extracting this quantitative information from images offers many advantages for better analyzing data beyond only inspection. What information do images contain? What limitations do the data and extraction methods possess? A range of methods exist for extracting intensity, size, number, absolute and relative morphology, colocalization and motion measurements. Manual, threshold-based and advanced segmentation methods all have a place and the optimal balance between manual intervention and automation varies with scale and type of data. These general core methods provide a diverse array of tools that can serve many tasks. Specific analysis implementations offer advantages in some cases using particular object morphologies or other 
assumptions about the images. Correct use of any processing steps is essential for meaningful quantification.

When all stages of image processing and analysis are appropriately put together, the capabilities of microscopy are extended far beyond solely obtaining visual representation of objects. It is therefore worthwhile to understand the tools available to achieve more complete analysis of images.

Figure 1. Challenges of dealing with image data and some of the solutions and methods that will be discussed and exemplified.

\begin{tabular}{|l|l|}
\hline \multicolumn{1}{|c|}{ Challenges } & \multicolumn{1}{|c|}{ Solutions } \\
\hline $\begin{array}{l}\text { Images are in specific and uncommon formats. } \\
\text { "Double-clicking on my computer doesn't } \\
\text { open my image". }\end{array}$ & $\begin{array}{l}\text { Specific software such as FIJ/ImageJ and } \\
\text { many others to open, process visualize and } \\
\text { quantify image data. }\end{array}$ \\
\hline $\begin{array}{l}\text { High-bit depth images and incompletely filled } \\
\text { dynamic range }\end{array}$ & $\begin{array}{l}\text { Digital contrast changes - optimal display and } \\
\text { conversion. }\end{array}$ \\
\hline $\begin{array}{l}\text { Data has more than 2 Dimensions - z-stacks, } \\
\text { time-series, z-stack time-series . . }\end{array}$ & $\begin{array}{l}\text { 3D and time series display and analysis. Stacks } \\
\text { and hyper-stacks. Image projection display. }\end{array}$ \\
\hline $\begin{array}{l}\text { Channels are pseudo-colors and go beyond R, } \\
\text { G and B }\end{array}$ & $\begin{array}{l}\text { Composite channels. Look-Up Tables. Color } \\
\text { space models. }\end{array}$ \\
\hline Large amounts of data & Cloud and local hardware options. \\
\hline Low signal to noise ratio & $\begin{array}{l}\text { Appropriate image processing before } \\
\text { presentation and quantification. }\end{array}$ \\
\hline Ethics: Not accidentally changing your data & Understanding of all processing steps. \\
\hline Showing and sharing data & $\begin{array}{l}\text { Appropriate formats for the relevant media } \\
\text {-presentation, print, web . . }\end{array}$ \\
\hline Obtaining accurate numbers from images & $\begin{array}{l}\text { Accurate capture, faithful processing, careful } \\
\text { quantification. Knowledge of the limitations. }\end{array}$ \\
\hline
\end{tabular}

